\title{
BMJ Open Oral glucocorticoid use and \\ osteonecrosis in children and adults with chronic inflammatory diseases: a population-based cohort study
}

\author{
Daniel B Horton, ${ }^{1}$ Kevin Haynes, ${ }^{2}$ Michelle R Denburg, ${ }^{3}$ Mihir M Thacker, ${ }^{4}$ \\ Carlos D Rose, ${ }^{5}$ Mary E Putt, ${ }^{6}$ Mary B Leonard, ${ }^{7}$ Brian L Strom ${ }^{6,8}$
}

To cite: Horton DB, Haynes K, Denburg MR, et al. Oral glucocorticoid use and osteonecrosis in children and adults with chronic inflammatory diseases: a population-based cohort study. BMJ Open 2017;7:e016788. doi:10.1136/ bmjopen-2017-016788

\section{- Prepublication history and} additional material for this paper are available online. To view these files please visit the journal online (http://dx.doi. org/10.1136/bmjopen-2017016788).

Received 9 March 2017 Revised 12 June 2017 Accepted 22 June 2017

For numbered affiliations see end of article.

Correspondence to Dr Daniel B Horton; daniel. horton@rutgers.edu

\section{ABSTRACT}

Objectives We studied oral glucocorticoids and osteonecrosis, a rare but serious bone disease, in individuals with various chronic inflammatory diseases. We hypothesised that we would find stronger associations in adults versus children and in people with autoimmune diseases.

Design Retrospective cohort study.

Setting Population-representative data (1994-2013) from general practices in the UK.

Participants Children and adults diagnosed with asthma; inflammatory bowel disease; juvenile, psoriatic or rheumatoid arthritis; psoriasis; or systemic lupus.

Exposures Oral glucocorticoid patterns.

Primary and secondary outcome measures Diagnosed osteonecrosis (primary) and osteonecrosis plus clinical features (eg, symptoms, pain medication, surgical repair) (secondary). Discrete time failure models estimated the adjusted hazard ratio (aHR) of incident osteonecrosis following oral glucocorticoid exposure. Hypothesis testing was one sided (with corresponding $90 \% \mathrm{Cl}$ ) since glucocorticoids were unlikely protective.

Results After adjusting for demographic, disease-related and health utilisation factors, glucocorticoid exposure was associated with osteonecrosis in adults (ages 18-49, aHR $2.1(90 \% \mathrm{Cl} 1.5$ to 2.9$)$; ages $\geq 50$, aHR $1.3(90 \%$ Cl 1.01 to 1.7)). However, low-dose glucocorticoids, corresponding to average doses $<7.5 \mathrm{mg}$ prednisolone daily and maximum doses $<30 \mathrm{mg}$ daily, were not associated with osteonecrosis in adults. Furthermore, even at high glucocorticoid doses, there was no evidence of increased osteonecrosis among glucocorticoid-exposed children ( $\mathrm{p}=0.04$ for interaction by age) (any glucocorticoid exposure, ages 2-9: aHR 1.1 (90\% Cl 0.7 to 1.7); ages 1017: aHR 0.6 (90\% Cl 0.3 to 1.6)). Arthritis, inflammatory bowel disease and lupus were independently associated with osteonecrosis, but there was a similar dose relationship between glucocorticoids and osteonecrosis among adults with low-risk and high-risk diseases.

Conclusions Glucocorticoid use was clearly associated with osteonecrosis in a dose-related fashion in adults, especially young adults, but this risk was not detectable in children. The absolute risk of glucocorticoid-associated osteonecrosis in the general paediatric population and in adults taking low glucocorticoid doses is at most extremely small.
Strengths and limitations of this study

- Unlike most other studies of glucocorticoids and osteonecrosis performed in specialty centres, a population-based study allowed for comparison across a wide range of ages and diseases and limited bias from selective inclusion of people with the most severe disease and had better generalisability to the general population.

- This is the largest paediatric study of glucocorticoids and osteonecrosis performed to date.

- The primary outcome definition has not been validated, but the secondary outcomes supported the main findings

- We could not eliminate the possibility of bias from residual confounding.

- Statistical power to detect an association in children was somewhat limited.

\section{INTRODUCTION}

Systemic glucocorticoids are commonly used to treat chronic inflammatory diseases such as asthma, inflammatory bowel disease (IBD) and rheumatoid arthritis (RA). Among many glucocorticoid-associated toxicities, osteonecrosis is a rare, but potentially disabling, condition of bones and joints. ${ }^{1}$ Also called avascular necrosis, osteonecrosis results from local trauma or systemic factors that impair blood flow and cause bone cell death in susceptible individuals. ${ }^{2}$ The pathophysiology of glucocorticoid-associated osteonecrosis is complex and multifactorial. ${ }^{2}$ Large osteonecrosis lesions can compromise quality of life and sometimes necessitate joint repair or replacement. ${ }^{3}$

Most studies on glucocorticoids and osteonecrosis in adults have focused on heavily glucocorticoid-exposed individuals with highrisk diseases such as leukaemia and systemic lupus erythematosus (SLE). ${ }^{4} 5$ Few population-based studies have been performed. 
One US study showed a link between brief glucocorticoid courses and osteonecrosis, with rising risk after multiple courses. ${ }^{6}$ However, this study did not adjust for any confounders, even age, sex or underlying disease. Another, more sophisticated UK population-based study of adults reported that oral glucocorticoids and diseases they treat (eg, cancer, connective tissue diseases) were independently associated with osteonecrosis. ${ }^{7}$ This study did not examine glucocorticoid dose or duration in detail or whether certain exposure levels may not predispose to osteonecrosis. Two Chinese population-based studies also implicated glucocorticoid exposure as a strong risk factor for femoral head osteonecrosis. ${ }^{8}{ }^{9}$ However, one study analysed binary, self-reported glucocorticoid intake ${ }^{8}$; the other included only individuals with osteonecrosis in nine tertiary care hospitals, limiting interpretability of its findings. ${ }^{9}$

Even less is known about glucocorticoids and osteonecrosis in children besides those with leukaemia, SLE or transplants. Unlike adults, children may have more protection from glucocorticoid-associated bone damage because of increased skeletal (re)modelling. ${ }^{10}$ Indeed, one population-based study of hip osteonecrosis in young children (Perthes disease) suggested that systemic glucocorticoid exposure was not a risk factor in children with asthma. ${ }^{11}$ However, this study did not examine drug timing or dosage, leaving unanswered questions about the risk of high-dose exposure and about other indications for glucocorticoid use. Furthermore, this study excluded older adolescents, a group at higher risk for glucocorticoid-associated osteonecrosis with certain highrisk diseases (eg, leukaemia and SLE). ${ }^{12}{ }^{13}$ Nonetheless, such high-risk diseases can themselves adversely affect bone health and have unique treatments (including high-intensity glucocorticoid regimens), potentially limiting generalisability to other paediatric populations. We hypothesised that glucocorticoid exposure was associated with osteonecrosis in a dose-dependent fashion in people with chronic inflammatory diseases, with stronger effects in adults than in children (as the capacity for skeletal remodelling decreases) and in people with autoimmune diseases.

\section{METHODOLOGY \\ Design}

We conducted a retrospective cohort study of data from 1994 to 2013 using The Health Improvement Network (THIN), a population-representative database with electronic health records of $>11$ million patients from general practices across the UK. ${ }^{14}$ THIN uses Vision software to collect anonymised practice-level and subject-level data during routine care, including demographics, diagnoses, outpatient prescriptions and specialist and hospital referrals. In the UK, subspecialists function as consultants, and general practitioners prescribe almost all outpatient medications with the exception of most biologic and parenteral medications. THIN is a widely used data source for pharmacoepidemiologic research ${ }^{15}$ and has been used to study oral glucocorticoids ${ }^{16}$ and osteonecrosis. $^{7}$

\section{Ethics approval}

This study was declared exempt from review by the institutional review boards of the University of Pennsylvania (\#818082) and Rutgers Biomedical and Health Sciences (Pro20150002631). This study was approved by THIN's scientific review committee (\#13-043).

\section{Subject selection}

Eligible subjects were at least 2 years old, registered in practices with Vision software and had a diagnosis of asthma, psoriasis, autoimmune arthritis (juvenile idiopathic arthritis (JIA), psoriatic arthritis (PsA) or RA), IBD or SLE. Diagnosis of an inflammatory disease of interest was determined by Read codes (analogous to ICD-9/10 codes) for conditions previously validated in THIN or other similar UK databases (see supplementary file 1 for complete list). ${ }^{17-22}$ We focused on people with chronic inflammatory diseases to help control for the indication for glucocorticoid use and reduce bias from confounding by indication. Inclusion of individuals with a diagnosis of asthma and psoriasis also required use of a bronchodilator and topical vitamin $\mathrm{D}$ analogue, respectively, to select for patients with more certain and severe illness. Children under the age of 2 were excluded because these diseases are uncommon and more likely misdiagnosed at such young ages. People with congenital hip dysplasia, Gaucher disease, sickle cell disease or prior osteonecrosis, cancer (other than non-melanoma skin cancer), decompression sickness or hematopoietic or solid organ transplant were excluded to limit confounding from these conditions at particularly high risk for osteonecrosis.

\section{Exposures}

The index date was the first day after a 183-day glucocorticoid-free baseline period, starting with the latter of THIN registration or Vision software initiation (supplementary figure S1). The 6-month baseline interval, a common practice in pharmacoepidemiologic studies, was based on prior research showing that incidence rates of most conditions approach expected rates by 6 months after registration. ${ }^{23}$ Subjects prescribed glucocorticoids during the baseline period were excluded (new-user design). Subjects were considered unexposed until their first glucocorticoid prescription. Exposed subjects were considered exposed through the end of the study period, since osteonecrosis may be diagnosed many years after glucocorticoid exposure. ${ }^{24}$

We characterised oral glucocorticoid prescriptions by glucocorticoid type, prednisone-equivalent dose and duration. Prescriptions written within 12 weeks of the scheduled completion date of prior prescriptions were considered part of the same course, to account for unwritten tapering and skipped doses. ${ }^{16}$ To estimate the relative hazards of oral glucocorticoid exposure across the age spectrum, we categorised observed exposure levels of dose and duration as low, medium or high 
based on tertiles of age-specific distributions in the study population (supplementary figure S2). Exposures were evaluated across several models of duration and dosage: duration (days), cumulative dose (mg), mean dose (mg/ day), maximum dose $(\mathrm{mg} /$ day) and dose intensity within 1 and 4 months of first prescription dose $(\mathrm{mg} / \mathrm{kg} /$ day).

\section{Outcomes}

Incident osteonecrosis was defined by the first osteonecrosis diagnosis with Read codes previously used in THIN and similar UK databases (supplementary table S2) ${ }^{725}$ Since these codes have not been formally validated, secondary analyses identified people with osteonecrosis codes and associated clinical features, such as joint pain, pain medication use and subsequent surgical repair. Subjects were censored for exclusion diagnoses, end of follow-up in THIN or death. Of note, we included as outcomes diagnoses of Perthes disease, the most common form of childhood osteonecrosis. While Perthes disease is generally thought to occur independently of glucocorticoid exposure, ${ }^{11}$ we did not presume that all cases of osteonecrosis in younger children were truly idiopathic and unrelated to glucocorticoid exposure; young children can be diagnosed with glucocorticoid-associated hip osteonecrosis even at young ages typically associated with Perthes disease. ${ }^{26}$

\section{Covariates}

Covariates assessed included demographics, medical and musculoskeletal comorbidities (eg, thrombotic disorders, fractures, osteoarthritis), other medication use, health utilisation indices (eg, number of medications, office visits) and lifestyle factors (eg, alcohol, smoking), chosen based on prior literature and clinical experience. Number of prior visits and medications was collected during the 183-day baseline period. All other covariates were collected using all available preindex data, a strategy that is comparable with confounding adjustment over fixed intervals. ${ }^{27}$ Four age categories $(2-9,10-17,18-49$ and $\geq 50$ years) were chosen based on age-based changes in skeletal structure and baseline incidence of osteonecrosis. $^{725}$

\section{STATISTICAL ANALYSIS \\ Association between glucocorticoid exposure and osteonecrosis}

The association between glucocorticoid exposure and osteonecrosis was estimated using discrete time failure modelling. ${ }^{28}$ Functionally similar to Cox regression, discrete time failure modelling is an alternative, more flexible time-to-event approach. These models compare the rate of an outcome between exposed and unexposed individuals followed across successive time periods (risk sets) until censoring or outcome. ORs are estimated over each consecutive risk set and then pooled to generate an HR. This approach was chosen over Cox regression for its computational efficiency and because it more easily handles ties and non-proportional hazards (as seen with time-varying exposures). ${ }^{28} 29$ The primary analysis compared time-updating glucocorticoid exposure to no exposure across 1-year intervals, applying variance adjustment for practice due to among-practice heterogeneity in a variety of clinical factors, including study variables (supplementary table S3). ${ }^{30}$ Multivariable models were derived using purposeful backwards selection of all covariates. ${ }^{31}$ While such an approach has its limitations, ${ }^{32}$ it allowed us to consider a large number of clinically plausible potential confounders and achieve a parsimonious model for a rare outcome. The final model contained predetermined confounders (age, sex and inflammatory disease) and covariates that changed HR by $>10 \%$ or had $\mathrm{p}$ value $<0.05$. Covariates were ascertained before the index date and updated for exposed individuals at the time of the first oral glucocorticoid prescription of the study period. Variables missing $\geq 10 \%$ of values were not included in multivariable models except in sensitivity analyses (see below). All multivariable models included the interaction of age and sex due to well-established sex-specific differences in the rates of osteonecrosis in children and adults. ${ }^{733}$

\section{Association between glucocorticoid dose/duration and osteonecrosis}

To evaluate dose or duration response, we calculated HRs of osteonecrosis at medium and high exposure levels compared with low-level exposure. We secondarily compared different exposure levels to the unexposed, to determine which specific dose levels conferred increased osteonecrosis rates. We evaluated age category and inflammatory disease diagnosis for effect modification; $\mathrm{p}$ values $<0.1$ by likelihood ratio testing were considered supportive of effect modification. All dose models were prespecified and represented different hypotheses about the relationship between glucocorticoid exposure and osteonecrosis rates. We hypothesised that certain dose models (ie, dose intensity) would yield stronger effect sizes than others. For these reasons, we did not adjust for multiple comparisons. Whereas younger children and older children/adolescents were considered separately in analyses of any glucocorticoid exposure, we combined paediatric patients into one cohort to analyse the effects of glucocorticoid dose. This allowed us to pool our limited number of events within each dose category across paediatric age groups.

\section{Model-derived subgroup-specific incidence rates of osteonecrosis}

The expected incidence of osteonecrosis was standardised for subgroups of interest using predictive margins. ${ }^{34}$ In short, we assigned duplicate datasets of the entire cohort to be alternatively unexposed or exposed to oral glucocorticoids. We then generated expected values (hazards) for the outcome in the hypothetical populations through regression using the original data. These expected values were used to generate model-derived incidence rates of osteonecrosis for groups of different ages, sexes and diagnoses, with or without glucocorticoid exposure. We 
used resampling methods to generate CIs, bootstrapping across 400 iterations, a number based on prior methodologic and applied studies. ${ }^{35} 36$

\section{Sensitivity analyses}

Various sensitivity analyses evaluated the impact of model assumptions and potential sources of bias. We examined alternative dose models, including age-based/ weight-based dose categories, analyses of the most recent glucocorticoid course, analyses with shorter windows of exposure after glucocorticoids (5, 3 and 2 years) and analyses considering hospitalisation within 1 month of an oral glucocorticoid prescription as a source of unrecorded high-dose (possibly parenteral) glucocorticoid exposure. We also evaluated hospitalisation as a potential effect modifier between glucocorticoids and osteonecrosis. We performed an analysis of children with osteonecrosis outcomes other than Perthes disease (ie, starting after age 14 or affecting joints other than the hip). We examined the impact of missing confounder data by using multiple imputation with 20 imputed datasets. ${ }^{37}$ We examined the potential impact of residual confounding by the rule-out method. ${ }^{38}$ This approach tests whether a theoretical confounder could nullify results over a range of prevalence and different associations with the exposure and the outcome.

We used one-sided hypothesis tests for the association of glucocorticoids and osteonecrosis since a finding of a protective effect of glucocorticoids lacked scientific credibility. Hypotheses related to other variables (eg, inflammatory disease) were two sided. We used a type I error rate of 0.05 . To be consistent with the sidedness of hypothesis testing, we used a $90 \%$ two-sided CI for the primary outcome and a 95\% two-sided CI for all other estimates. All analyses were performed using Stata 12.1 (Stata Corp, College Station, Texas, USA).

\section{RESULTS}

\section{Characteristics of the study population}

The study cohort consisted of 920321 individuals followed for 6.8 million person-years (supplementary figure S3). Of these, 263037 (28.6\%) were prescribed oral glucocorticoids during the study period (supplementary figure S3, supplementary table S4). One quarter entered the study before age $18(225936,24.5 \%)$ (table 1). Asthma was the most common inclusion diagnosis $(756718,82.2 \%)$, while 205747 people (22.4\%) had another chronic inflammatory disease of interest. Compared with unexposed patients, glucocorticoid-exposed patients were generally older at baseline with a higher prevalence of all inclusion diagnoses except psoriasis. Exposed patients also had more medical and musculoskeletal comorbidities and more medication and healthcare utilisation.

Osteonecrosis developed in 428 individuals. Overall rates of osteonecrosis were highest among young children (table 2). The proportion of osteonecrosis involving weight-bearing joints (most commonly the hip) and with recorded symptoms was similar between cohorts. However, exposed patients were more likely to receive pain medications $(\mathrm{p}<0.001)$ and subsequently undergo surgical joint repair $(\mathrm{p}=0.03)$. Among patients with osteonecrosis, the vast majority $(410,95.8 \%)$ met at least one secondary outcome definition, suggesting that study outcome definitions were valid and clinically relevant.

\section{Association of oral glucocorticoids and osteonecrosis}

Age significantly modified the relationship between oral glucocorticoids and osteonecrosis $(\mathrm{p}=0.04$ for interaction). After adjusting for age and sex, inflammatory disease, other rheumatic diseases, fracture, calendar year and number of prescribed medications, osteonecrosis developed in glucocorticoid-exposed adults more than unexposed adults (ages 18-49: aHR 2.1, $90 \%$ CI 1.5 to 2.9; ages $\geq 50$ :aHR $1.3,90 \%$ CI 1.01 to 1.7 ) (table 3 ). Non-oral formulations of glucocorticoids were not confounders or independently associated with osteonecrosis. Analyses of secondary outcomes (eg, osteonecrosis plus recorded symptoms, pain medication use or surgical repair) were highly consistent (supplementary table S5).

In terms of exposure level, adults ages 18-49 exposed to high-dose versus low-dose glucocorticoids showed substantially higher risk of osteonecrosis (aHRs 1.4-4.5) (figure 1). A dose response was also evident among adults ages $\geq 50$ across several models. Compared with no exposure, rates of osteonecrosis were particularly elevated in adults ages $<50$ years exposed to sustained, high-dose glucocorticoids (high 4-month dose intensity: aHR 6.8, 90\% CI 4.1 to 11.2) (supplementary table S6). Notably, among younger adults, both short and long glucocorticoid courses were associated with osteonecrosis. In contrast, low-dose glucocorticoid exposure (including mean doses $<7.5 \mathrm{mg} /$ day and maximum doses $<30 \mathrm{mg} /$ day) was not associated with osteonecrosis in adults (aHRs $0.8-1.4, \mathrm{p}>0.05)$.

In contrast, there was no suggestion of an association between glucocorticoids and osteonecrosis in children (ages 2-9: aHR 1.1, 90\% CI 0.7 to 1.7; ages 10-17: aHR $0.6,90 \%$ CI 0.3 to 1.6 ) (table 3 ). In the paediatric population, there was no overall risk or any apparent dose response; children exposed to high-dose glucocorticoids did not experience elevated rates of osteonecrosis (aHRs $0.7-1.3, \mathrm{p}>0.05)$.

When evaluating the risk of different inflammatory diseases, people diagnosed with autoimmune arthritis, IBD and SLE had higher rates of osteonecrosis than people with asthma (table 3). However, after accounting for differences in baseline osteonecrosis rates, underlying disease did not modify the effect of glucocorticoid dose ( $p>0.1$ for interaction for all dose models); regardless of inflammatory disease diagnosis, we observed a similar dose relationship between glucocorticoids and osteonecrosis (supplementary figure S4).

With regard to absolute osteonecrosis rates, demographics and autoimmune disease diagnosis were important correlates. Within each age group, having a 
Table 1 Characteristics of study population

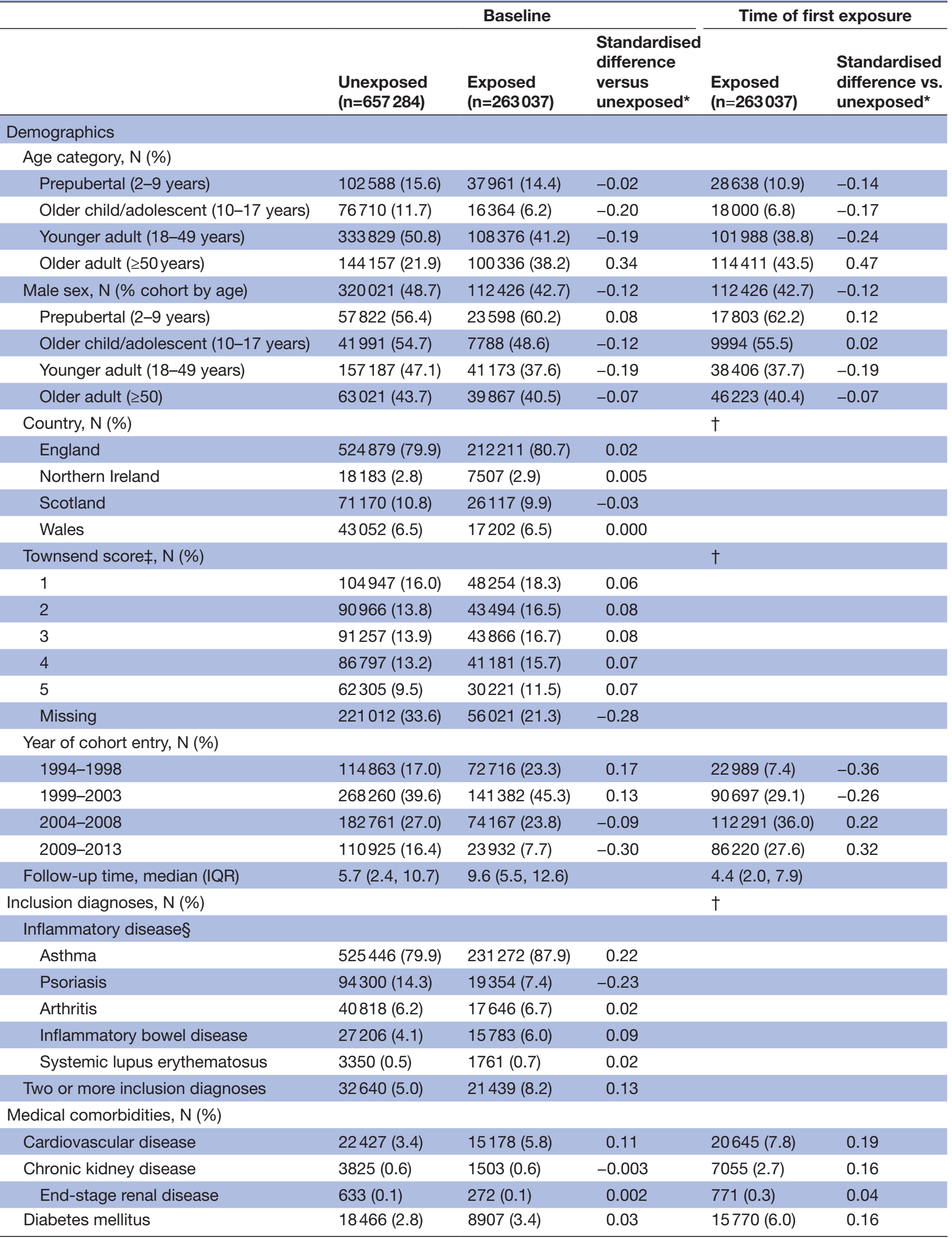

Continued 
Table 1 Continued

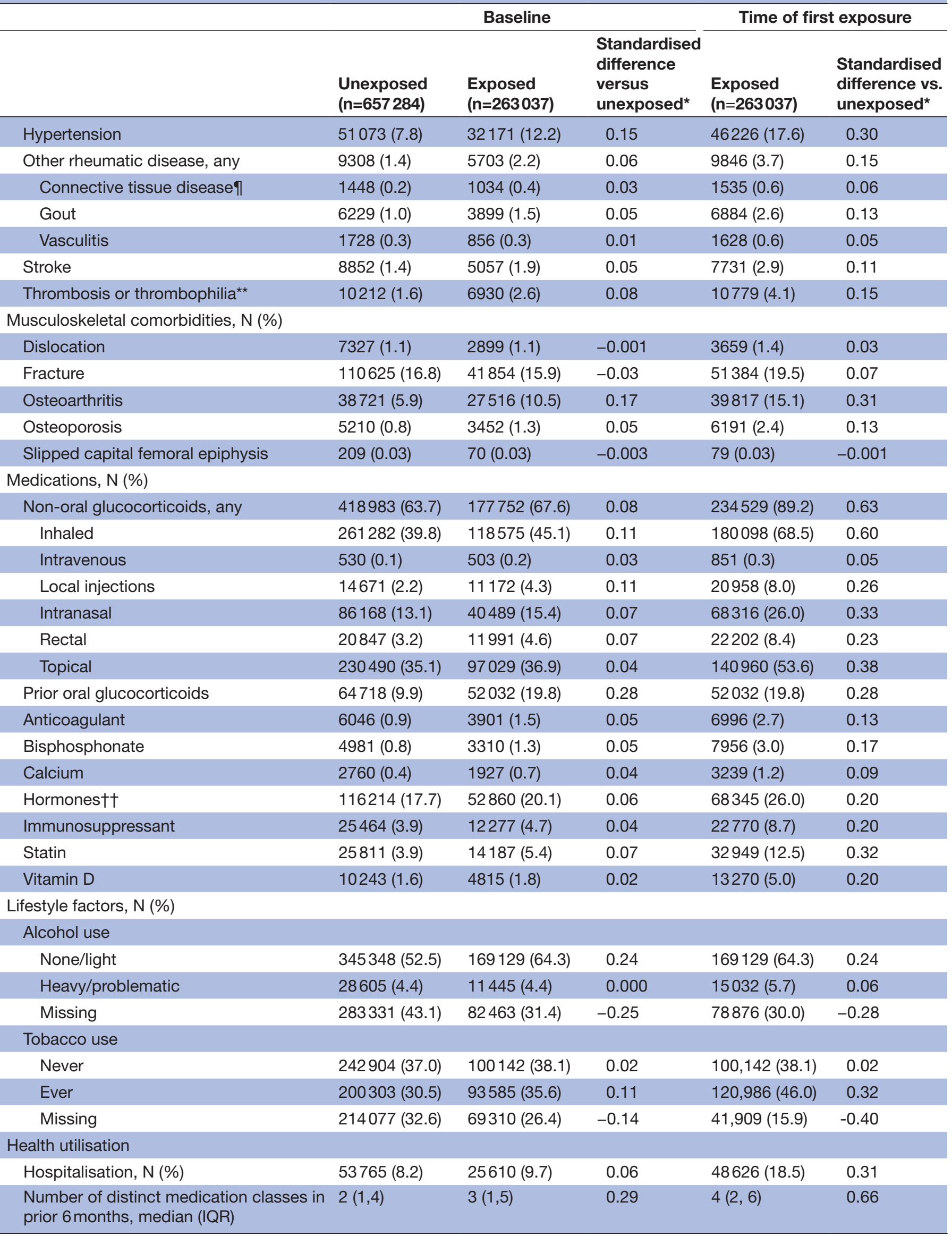




\begin{tabular}{|c|c|c|c|c|c|}
\hline & \multicolumn{3}{|c|}{ Baseline } & \multicolumn{2}{|c|}{ Time of first exposure } \\
\hline & $\begin{array}{l}\text { Unexposed } \\
(n=657284)\end{array}$ & $\begin{array}{l}\text { Exposed } \\
(n=263037)\end{array}$ & $\begin{array}{l}\text { Standardised } \\
\text { difference } \\
\text { versus } \\
\text { unexposed* }^{*}\end{array}$ & $\begin{array}{l}\text { Exposed } \\
(n=263037)\end{array}$ & $\begin{array}{l}\text { Standardised } \\
\text { difference vs. } \\
\text { unexposed }^{*}\end{array}$ \\
\hline $\begin{array}{l}\text { Number of outpatient encounters in prior } \\
6 \text { months, median (IQR) }\end{array}$ & $2(0,4)$ & $2(1,4)$ & 0.08 & $5(2,9)$ & 0.39 \\
\hline
\end{tabular}

*Standardised differences $>0.1$ or $<-0.1$ suggest imbalance. $\dagger$ Not updated at time of exposure; same as baseline values.

łLocal measures of deprivation, ranging from 1 (lowest local deprivation) to 5 (highest local deprivation).

§Some patients had more than one diagnosis.

ПConnective tissue diseases other than systemic lupus, including Sjögren syndrome, systemic sclerosis, mixed connective tissue disease, dermato/polymyositis.

${ }^{* \star}$ Not including stroke.

††Hormonal contraceptives or hormone replacement therapy.

$\ddagger \ddagger$ Including only specialists who manage chronic inflammatory diseases of interest.

disease such as arthritis, IBD or SLE was associated with a larger increase in osteonecrosis incidence (relative to asthma) than exposure to glucocorticoids (relative to no exposure). Per 100000 person-years, glucocorticoid exposure among men under age 50 was associated with 4.0 (90\% CI 2.4 to 5.8) additional cases of osteonecrosis for those with asthma or psoriasis (number needed to harm $(\mathrm{NNH}) \approx 25000)$ and $10.6(90 \%$ CI 6.0 to 15.3 ) additional cases for those with arthritis, IBD or SLE $(\mathrm{NNH} \approx 9430)$ (table 4). Compared with the unexposed, glucocorticoid-exposed women and older men had small absolute increases in osteonecrosis rates (range 1-5/100 000 person-years, NNH $21000-83000$ ).

\section{Sensitivity analyses}

When considering shorter exposure windows after glucocorticoids (2-5 years), results were consistent with analyses using unbounded windows (supplementary figures S5 and S6). These findings suggest that rates of newly diagnosed osteonecrosis remain similar for many years after glucocorticoid exposure. Analysis using multiple imputation to incorporate socioeconomic status (30.1\% missing data) and alcohol abuse (39.7\% missing data) was no different from the primary analysis (supplementary table S7). Analyses incorporating weight into dose level or equating hospitalisation with high-level exposure were also similar (supplementary table S8). Analyses of the most recent glucocorticoid course showed weaker effects than cumulative glucocorticoid exposure, underscoring the impact of repeated courses (supplementary table S8). Hospitalisation did not modify the effect of glucocorticoids on osteonecrosis (test for interaction $p>0.1$ ). Analyses of children after excluding those with presumed Perthes disease were similar (ages 2-9: aHR $1.4,90 \%$ CI 0.2 to 9.8 ; ages $10-17$ : aHR $0.8,90 \%$ CI 0.3 to 2.1 ) but limited by small sample size. Only strong unmeasured confounders (OR $\geq 4$ with glucocorticoid exposure and osteonecrosis, prevalence $\geq 15 \%$ ) could explain HR 2.1 among younger adults.

\section{DISCUSSION}

Among nearly 1 million people with chronic inflammatory diseases, we found that adults exposed to oral glucocorticoids developed osteonecrosis in a dose-dependent fashion. This association was most pronounced in adults under age 50 and in those taking higher-dose glucocorticoids over several months. In contrast, no association or dose relationship was apparent in children or adolescents prescribed oral glucocorticoids. Furthermore, even among adults, low-dose glucocorticoid exposures, corresponding to average daily doses $<7.5 \mathrm{mg}$ and maximum daily doses $<30 \mathrm{mg}$, were not associated with osteonecrosis. Regardless of age, autoimmune diseasespecifically, arthritis (JIA, PsA or RA), IBD and SLE-was an independent risk factor for osteonecrosis. Nonetheless, glucocorticoid dose appears to magnify the rate of osteonecrosis similarly for people with both low-risk (asthma, psoriasis) and high-risk diseases (arthritis, IBD, SLE).

To our knowledge, this is the largest paediatric study of glucocorticoids and osteonecrosis to date. The absence of any apparent relationship between glucocorticoids and osteonecrosis in children with chronic inflammatory diseases is a reassuring finding for the many young people taking oral glucocorticoids for such conditions. ${ }^{39}$ In part, this may reflect the unique pathophysiology of paediatric hip osteonecrosis (Perthes disease). ${ }^{11} 25$ Additionally, children may be relatively protected from glucocorticoid-associated bone damage because of greater skeletal remodelling and plasticity. ${ }^{10}$ Our findings contrast with the high incidence of glucocorticoid-associated osteonecrosis in older children with leukaemia and lupus. ${ }^{13} 2640$ Such discrepancies may reflect unique disease-specific and treatment-specific insults to bony physiology and metabolism or the order-of-magnitude higher levels of glucocorticoids received by children with these conditions. $^{41} 42$

Many prior studies have shown dose-dependent increases in osteonecrosis at higher levels of exposure in 
Table 2 Osteonecrosis outcomes and clinical features

\begin{tabular}{|c|c|c|c|c|c|c|c|}
\hline & \multicolumn{3}{|c|}{$\begin{array}{l}\text { Unexposed } \\
(n=657284)\end{array}$} & \multicolumn{3}{|c|}{$\begin{array}{l}\text { Exposed } \\
(\mathrm{n}=263037)\end{array}$} & \multirow[t]{2}{*}{ p Value } \\
\hline & $\mathbf{N}$ & Rate/\% & $95 \% \mathrm{Cl}$ & $\mathbf{N}$ & Rate/\% & $95 \% \mathrm{Cl}$ & \\
\hline \multicolumn{8}{|c|}{ Osteonecrosis, N; incidence per 100000 person-years (95\% Cl) } \\
\hline Ages 2-9 & 73 & 14.7 & $(11.7$ to 18.4$)$ & 21 & 18.4 & (12.0 to 28.3$)$ & 0.36 \\
\hline Ages 10-17 & 28 & 3.9 & (2.7 to 5.6$)$ & 4 & 2.9 & (1.1 to 7.7$)$ & 0.62 \\
\hline Ages $18-49$ & 81 & 3.3 & (2.6 to 4.1 ) & 41 & 7.9 & (5.9 to 10.6$)$ & $<0.001$ \\
\hline Ages $\geq 50$ & 102 & 6.4 & (5.2 to 7.7$)$ & 78 & 10.8 & (8.6 to 13.5$)$ & 0.007 \\
\hline \multicolumn{8}{|c|}{ Secondary osteonecrosis outcomes, N (\% of cases in cohort) } \\
\hline Joint imaging ${ }^{*}$ & 122 & 43.0 & & 72 & 50.0 & & 0.17 \\
\hline $\begin{array}{l}\text { Imaging, orthopaedic visit, emergency room } \\
\text { visit or hospitalisation† }\end{array}$ & 218 & 76.8 & & 120 & 83.3 & & 0.12 \\
\hline Osteonecrosis symptomsł & 150 & 52.8 & & 76 & 52.8 & & 0.99 \\
\hline Pain medications $\ddagger \S$ & 198 & 69.7 & & 121 & 84.0 & & $<0.001$ \\
\hline Orthopaedic visitๆ & 185 & 65.1 & & 97 & 67.4 & & 0.65 \\
\hline Subsequent surgical joint repair ${ }^{\star \star}$ & 90 & 31.7 & & 61 & 42.4 & & 0.03 \\
\hline Any secondary outcome & 271 & 95.4 & & 139 & 96.5 & & 0.59 \\
\hline \multicolumn{8}{|l|}{ Joints with osteonecrosis, N (\% of cases in cohort) } \\
\hline Shoulder & 9 & 3.2 & & 2 & 1.4 & & 0.27 \\
\hline Wrist & 14 & 4.9 & & 2 & 1.4 & & 0.07 \\
\hline Hip & 204 & 71.8 & & 97 & 67.4 & & 0.34 \\
\hline Perthes disease†† & 79 & $76.2 \dagger \dagger$ & & 21 & $80.8 \ddagger \ddagger$ & & 0.62 \\
\hline Knee & 20 & 7.0 & & 12 & 8.3 & & 0.63 \\
\hline Other & 4 & 1.4 & & 3 & 2.1 & & 0.60 \\
\hline Not otherwise specified & 50 & 17.6 & & 37 & 25.7 & & 0.05 \\
\hline
\end{tabular}

${ }^{*}$ Documented as ordered or performed within 6 months before diagnosis.

†Documented imaging and/or other opportunities for imaging within 6 months before diagnosis.

$\neq$ Within \pm 1 year of diagnosis.

§Pain medications include prescribed nonsteroidal anti-inflammatories or opiates.

IWithin 6 months before diagnosis until any time afterwards.

${ }^{* \star}$ Any time after diagnosis; includes both joint-sparing and joint replacement procedures.

††Hip osteonecrosis diagnosed in children before age 15; percentage reflects osteonecrosis in paediatric cohort only.

adults, ${ }^{45}$ but it has been unclear whether a dose-threshold for osteonecrosis risk existed. In our study, low-dose glucocorticoids do not appear to increase rates of osteonecrosis. Our definition of low average daily dose was $<7.5 \mathrm{mg}$ /day; this corresponds well with low-dose thresholds considered elsewhere. ${ }^{43}$ Of note, the apparent safety of low-dose exposure with respect to osteonecrosis contrasts with data on osteoporosis: glucocorticoids increase fracture rates even at similarly low doses. ${ }^{44}$ Interestingly, unlike other exposure models, even short glucocorticoid courses were associated with increased rates of osteonecrosis among young adults in our study. Another study, though limited in its design and analyses, suggested a similar effect of short-course glucocorticoids. ${ }^{6}$ Perhaps some osteonecrosis-prone individuals may develop bone disease even after relatively short (and possibly higher dose) glucocorticoid exposures. This hypothesis bears further examination.

The predisposition of younger adults and strong association with high-dose intensity have been reported previously. ${ }^{45}$ However, the vast majority of prior studies focused on single disease populations with extensive glucocorticoid exposure, such as haematologic malignancies, transplantation and SLE. ${ }^{45}$ These studies have notable limitations: selection bias of individuals with particularly severe disease; ascertainment bias through proactive monitoring of treatment complications; confounding by indication, whereby individuals with more severe diseases receive more glucocorticoids, making it difficult to distinguish the effects of disease from medication; and questionable generalisability to other populations. In contrast, we studied a nationally representative sample of people diagnosed with various diseases (predominantly asthma, which is not associated with osteonecrosis ${ }^{71}$ ), yielding greater generalisability, less selection bias and less confounding by indication. For instance, we found similar dose relationships between glucocorticoid exposure and osteonecrosis in people with 'high-risk' diseases (eg, SLE and RA) and in people with asthma, in whom severe 
Table 3 Primary multivariable analysis of oral glucocorticoid exposure and osteonecrosis, stratified by age

HR, exposed versus unexposed

\begin{tabular}{|c|c|c|c|c|c|}
\hline Variables & $\begin{array}{l}\text { Exposed } \\
\text { Unexposed (N) }\end{array}$ & $\begin{array}{l}\text { Outcomes } \\
\text { (N) }\end{array}$ & Unadjusted HR (CI*) & Adjusted HR†, $\left(\mathrm{Cl}^{\star}\right)$ & p Value \\
\hline \multicolumn{6}{|c|}{ Glucocorticoid exposure } \\
\hline Ages $10-17$ years & $\begin{array}{l}18000 \\
76710\end{array}$ & $\begin{array}{r}4 \\
28\end{array}$ & $\begin{array}{l}0.7 \\
(0.3 \text { to } 1.7)\end{array}$ & $\begin{array}{l}0.6 \\
(0.3 \text { to } 1.6)\end{array}$ & 0.20 \\
\hline Ages $\geq 50$ years & $\begin{array}{l}114411 \\
144157\end{array}$ & $\begin{array}{r}78 \\
102\end{array}$ & $\begin{array}{l}1.6 \\
\text { (1.2 to } 2.0)\end{array}$ & $\begin{array}{l}1.3 \\
\text { (1.01 to } 1.7)\end{array}$ & 0.05 \\
\hline
\end{tabular}

Other model variables

Chronic inflammatory disease

Asthma (reference)§ 212939

$501635 \quad 192$

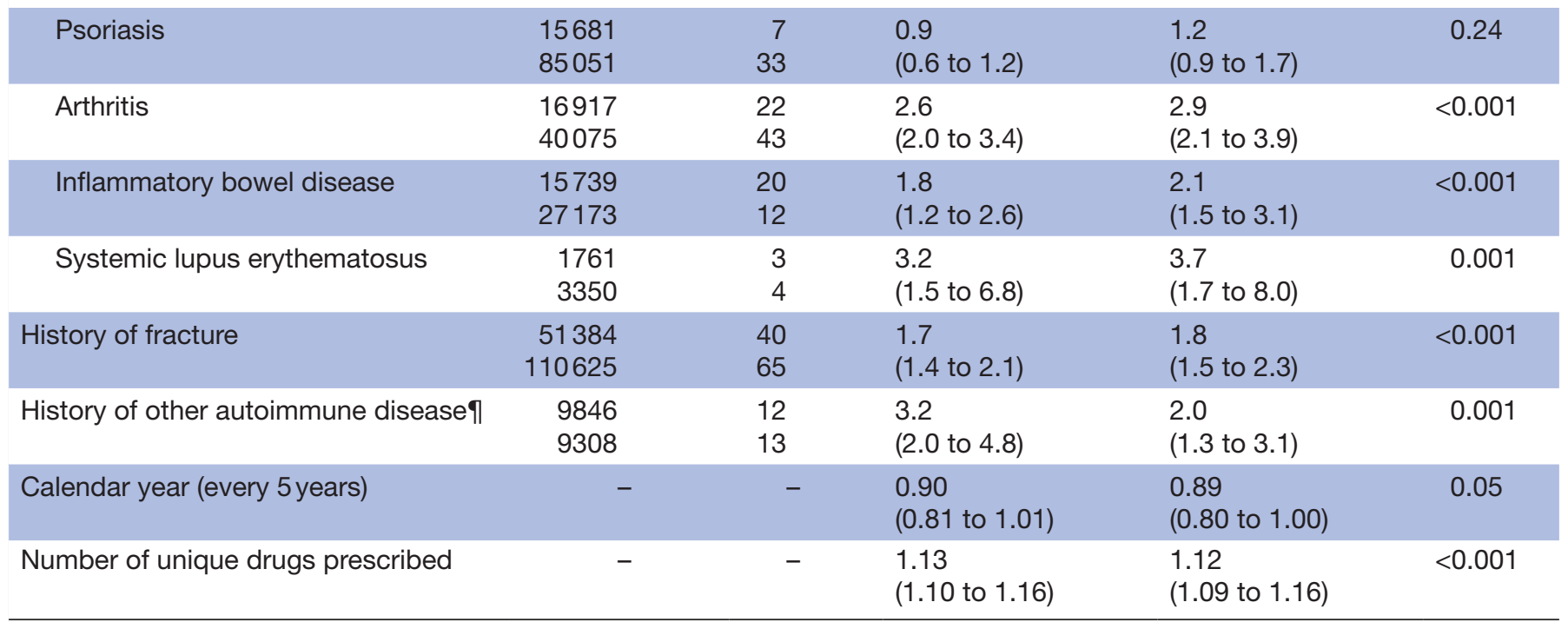

${ }^{*} 90 \% \mathrm{Cl}$ is shown for the association between oral glucocorticoid exposure and osteonecrosis. All other variables are shown with a $95 \% \mathrm{Cl}$. †Models adjusted for all variables shown and the interaction between sex and age.

$\ddagger$ One-sided $p$ values are shown for the association between oral glucocorticoid exposure and osteonecrosis. All other $p$ values are two sided. §Individuals with two or more chronic inflammatory diseases of inclusion were categorised as having the latter disease as listed here.

ๆAutoimmune diseases include connective tissue diseases other than systemic lupus (eg, Sjögren syndrome, systemic sclerosis), gout and vasculitis.

disease does not, to our knowledge, predispose to osteonecrosis. These findings suggest little impact of residual confounding by disease severity in our study. Furthermore, osteonecrosis incidence among patients diagnosed with SLE in our study was markedly lower than previously reported. ${ }^{478}$ In the context of the general population, this severe bone disease occurred rarely even in people diagnosed with high-risk autoimmune diseases. Overall, osteonecrosis rates in our population were comparable to other population-representative studies. ${ }^{725}$ Nonetheless, these previous studies did not dissect the complex relationships between age, glucocorticoid dose, inflammatory disease and osteonecrosis as we performed here. Osteonecrosis codes have not been validated in THIN, but the vast majority with osteonecrosis in our study had related clinical features (eg, symptoms, use of pain medication, subsequent operative repair), suggesting that we studied clinically relevant bone disease. While similar clinical sequelae could affect people with arthritis, the majority of subjects with osteonecrosis had asthma or psoriasis without arthritis. Furthermore, analyses of osteonecrosis with accompanying clinical features were highly consistent with primary results.

This study had potential limitations. In spite of considerable advantages of population-based research, generalisability to other geographic and ethnic populations could be limited. We may have overestimated drug exposure by studying glucocorticoids prescribed rather than taken and assuming non-overlapping courses; however, this would bias our results towards the null. Similar bias could 
Duration

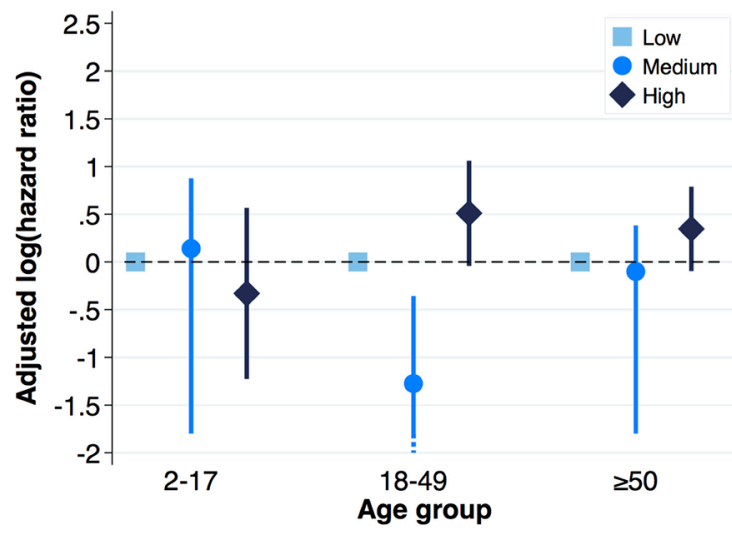

\section{Mean dose}

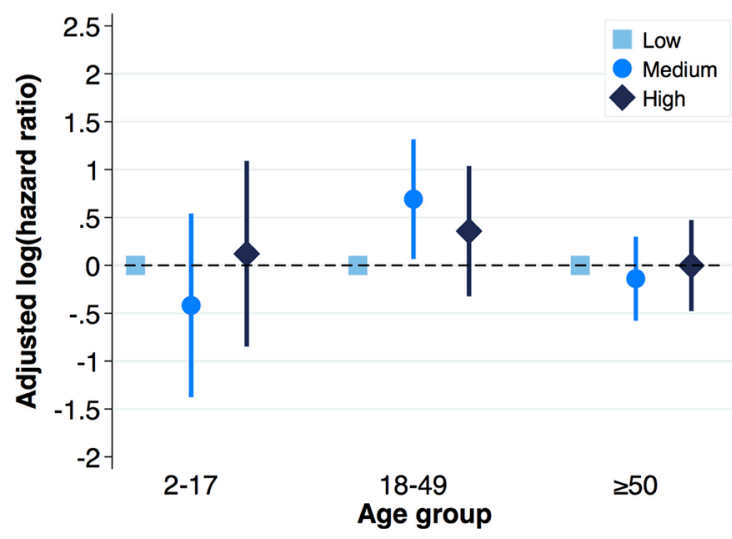

\section{Dose intensity, 1 month}

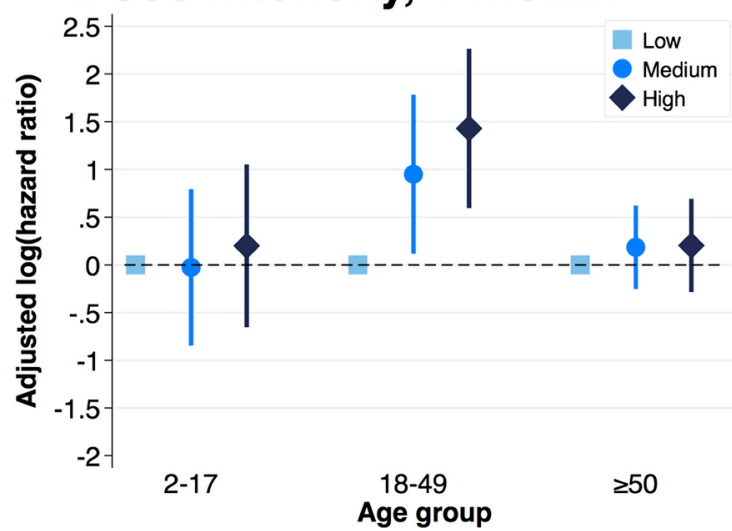

Cumulative dose

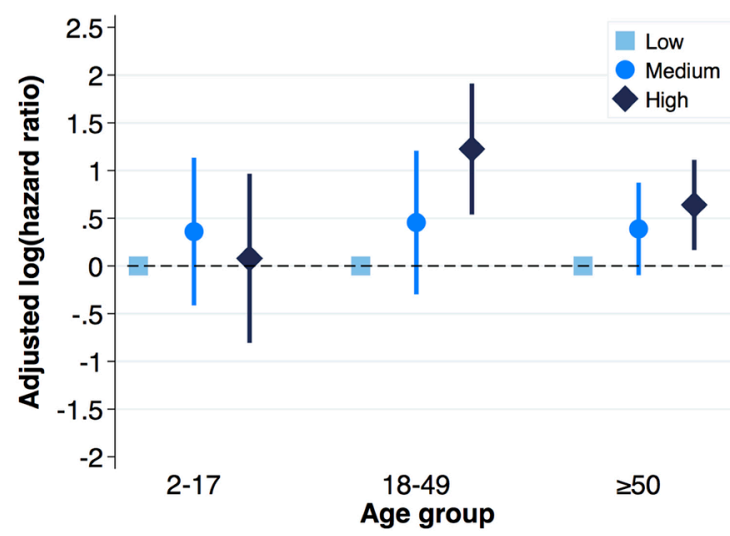

Maximum dose
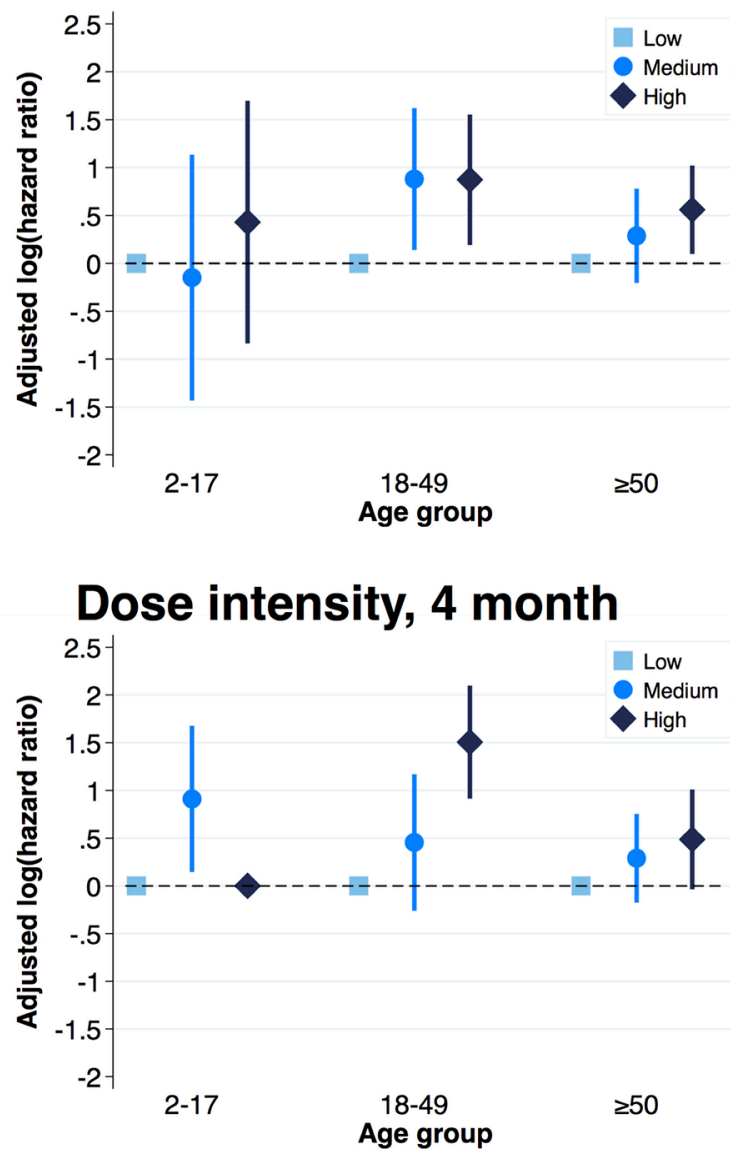

Figure 1 Age-stratified association between duration or dose of oral glucocorticoids and osteonecrosis. Analyses were limited to glucocorticoid-exposed patients, comparing medium-dose and high-dose to low-dose exposure (low-dose group shown with $\log (\mathrm{HR})=0$ for reference). Dose intensity represents the cumulative dose within 1 or 4 months of starting a new glucocorticoid course. Bars indicate $90 \% \mathrm{Cl}$.

result by assuming unbounded risk windows. However, analyses considering shorter exposure windows yielded similar results. While we allowed up to 12 weeks between prescriptions in the same course, many analyses (eg, any exposure, cumulative dose, mean dose or maximum dose) would not have changed with alternate, shorter windows. Osteonecrosis diagnosis has not been validated, and some individuals did not have prior imaging documented, so certain outcomes may have been misclassified. Nonetheless, age-specific and sex-specific incidence of osteonecrosis in our study was consistent with prior literature, and nearly all individuals diagnosed with osteonecrosis had documented clinical features compatible with this bone disease. Similarly, while inflammatory disease diagnosis may have been misclassified in some, certain autoimmune diseases remained independent risk factors of osteonecrosis, as expected. Furthermore, dose-response relationships between glucocorticoids and osteonecrosis were similar across diseases. 
Table 4 Model-derived, subgroup-specific incidence of osteonecrosis per 100000 person-years with $90 \% \mathrm{Cl}$

\begin{tabular}{|c|c|c|c|c|c|c|}
\hline & \multicolumn{3}{|c|}{ Female } & \multicolumn{3}{|c|}{ Male } \\
\hline & Unexposed & Exposed & Risk difference & Unexposed & Exposed & Risk difference \\
\hline \multicolumn{7}{|l|}{ Ages 2-9 } \\
\hline Asthma/psoriasis* & $(4.5,8.3)$ & $(3.9,9.0)$ & $(-2.1,1.7)$ & $(17.7,24.0)$ & $(14.5,25.5)$ & $(-6.2,5.3)$ \\
\hline Arthritis/IBD/SLE & 15.6 & 15.5 & -0.1 & 50.6 & 50.9 & -0.5 \\
\hline \multicolumn{7}{|l|}{ Ages 10-17 } \\
\hline \multirow[t]{2}{*}{ Asthma/psoriasis* } & 3.0 & 2.9 & -0.0 & 3.9 & 3.8 & -0.0 \\
\hline & $(1.9,3.9)$ & $(1.6,4.2)$ & $(-0.8,0.7)$ & $(2.7,4.9)$ & $(2.5,5.2)$ & $(-1.1,0.9)$ \\
\hline \multirow[t]{2}{*}{ Arthritis/IBD/SLE } & 7.5 & 7.4 & -0.1 & 10.2 & 10.0 & -0.1 \\
\hline & $(4.4,10.6)$ & $(4.4,11.4)$ & $(-2.2,1.9)$ & $(6.7,13.3)$ & $(6.3,13.8)$ & $(-3.0,2.5)$ \\
\hline \multicolumn{7}{|l|}{ Ages 18-49 } \\
\hline Asthma/psoriasis* & $(1.3,2.1)$ & $(2.7,4.3)$ & $(1.1,2.5)$ & $(3.2,4.4)$ & $(6.0,9.7)$ & $(2.4,5.8)$ \\
\hline \multirow[t]{2}{*}{ Arthritis/IBD/SLE } & 4.6 & 9.4 & 4.8 & 10.3 & 21.0 & 10.6 \\
\hline & $(3.4,5.7)$ & $(6.9,11.7)$ & $(2.7,6.6)$ & $(8.3,12.4)$ & $(15.8,26.8)$ & $(6.0,15.3)$ \\
\hline \multicolumn{7}{|l|}{ Ages $\geq 50$} \\
\hline \multirow[t]{2}{*}{ Asthma/psoriasis* } & 5.3 & 6.8 & 1.5 & 4.3 & 5.5 & 1.2 \\
\hline & $(4.4,6.2)$ & $(5.6,8.0)$ & $(0.3,2.8)$ & $(3.5,5.1)$ & $(4.5,6.8)$ & $(0.2,2.4)$ \\
\hline \multirow[t]{2}{*}{ Arthritis/IBD/SLE } & 13.5 & 17.4 & 3.7 & 11.0 & 13.8 & 3.0 \\
\hline & $(11.1,15.9)$ & $(14.2,20.7)$ & $(0.7,7.1)$ & $(8.9,12.7)$ & $(11.2,17.3)$ & $(0.5,6.0)$ \\
\hline
\end{tabular}

IBD, inflammatory bowel disease; SLE, systemic lupus erythematosus.

*Subjects categorised as having asthma or psoriasis did not also have diagnoses of juvenile, psoriatic or rheumatoid arthritis, IBD or SLE.

Additionally, some patients followed by subspecialists may not have had osteonecrosis diagnoses recorded in the database, possibly underestimating osteonecrosis incidence among those with uncommon diseases. In addition, few people exposed to glucocorticoids other than prednisolone/prednisone had osteonecrosis, making it difficult to evaluate the impact of glucocorticoid type. Despite adjustment for confounding, bias from residual confounding (eg, lifestyle, ancestry, genetics, unrecorded drug exposures) may persist. However, our analyses were robust to unmeasured confounding and various assumptions, including unrecorded intravenous glucocorticoids with hospitalisation. Finally, despite our large cohort, we may have captured too few outcomes among glucocorticoid-exposed youth and adults on low-dose glucocorticoids to detect small or delayed effects. Nonetheless, given our huge sample size, that is meaningful information by itself. With effect estimates close to the null and the low incidence rates of osteonecrosis, the absolute risk of glucocorticoid-associated osteonecrosis in the general paediatric population and in adults taking low glucocorticoid doses is at most extremely small.

In summary, oral glucocorticoid exposure is associated with incident, clinically relevant osteonecrosis in adults with chronic inflammatory diseases in a dose-dependent manner. This association is strongest among adults under the age of 50. Low-dose glucocorticoids, corresponding to doses below $7.5 \mathrm{mg}$ daily and maximum doses under $30 \mathrm{mg}$ daily, are not significantly associated with osteonecrosis. No association or dose response from glucocorticoids was seen in a large general paediatric cohort under age 18. Further work should validate these findings in other large paediatric populations and investigate whether glucocorticoids besides predniso(lo)ne confer increased risk of osteonecrosis, as in paediatric leukaemia. Autoimmune diseases such as IBD, juvenile arthritis, PsA and RA and lupus are independent risk factors for osteonecrosis, but there is a similar dose relationship between glucocorticoids and osteonecrosis among adults with low-risk and high-risk diseases. Therapies that counteract the risk of glucocorticoid-associated osteonecrosis remain unclear and warrant further study.

\section{Author affiliations}

${ }^{1}$ Department of Pediatrics, Rutgers Robert Wood Johnson Medical School, Rutgers Center for Pharmacoepidemiology and Treatment Science, Institute for Health, Health Care Policy and Aging Research, Rutgers School of Public Health, Rutgers Biomedical and Health Sciences, New Brunswick, New Jersey, USA

${ }^{2}$ HealthCore, Wilmington, Delaware, USA

${ }^{3}$ Division of Nephrology, Department of Pediatrics, The Children's Hospital of Philadelphia, Perelman School of Medicine, University of Pennsylvania, Philadelphia, Pennsylvania, USA

${ }^{4}$ Department of Orthopedics, Nemours A.I. duPont Hospital for Children, Thomas Jefferson University, Wilmington, Delaware, USA

${ }^{5}$ Division of Rheumatology, Department of Pediatrics, Nemours A.I. duPont Hospital for Children, Thomas Jefferson University, Wilmington, Delaware, USA 
${ }^{6}$ Department of Biostatistics and Epidemiology, Perelman School of Medicine, University of Pennsylvania, Philadelphia, Pennsylvania, USA

${ }^{7}$ Division of Nephrology, Departments of Pediatrics and Medicine, Stanford School of Medicine, Stanford University, Stanford, California, USA

${ }^{8}$ Rutgers Biomedical and Health Sciences, Newark, New Jersey, USA

Acknowledgements We thank A. Russell Localio for his help with data analysis and Molly E. Collins for her editorial assistance.

Contributors DBH conceived and designed the study, with input from the other authors. DBH and $\mathrm{KH}$ acquired the data. DBH analysed the data, with support from A. Russell Localio, MRD, MEP, MBL and BLS. All authors interpreted the data and contributed to the writing of the paper. DBH wrote the first draft of the paper. Molly E. Collins provided editorial assistance for the paper. All authors revised and approved the final version. CDR, MEP and BLS supervised the project. DBH, MRD, MBL, MEP and BLS obtained funding for the study.

Funding This project was supported by grants from the National Institutes of Health (grant number T32-GM075766 to BLS for DBH's training, F32-AR066461 and K23-AR070286 to DBH, K23-DK093556 to MRD, U54-HD086984 to MEP, K24-DK076808 to MBL) and The NephCure Foundation American Society of Nephrology Research Grant to MRD. The funders had no role in study design, data collection and analysis, decision to publish or preparation of the manuscript.

Competing interests $\mathrm{KH}$ is an employee of HealthCore, a wholly owned research subsidiary of Anthem; MRD has received grant funding from Genentech and Mallinckrodt Pharmaceuticals and has consulted for Infiniti Medical, outside the submitted work; CDR has received grant funding from GSK, outside the submitted work; MBL has consulted for Amgen and Novartis, outside the submitted work; BLS has received grant funding from AstraZeneca and Takeda and has consulted for Abbott, AbbVie, Amgen, AstraZeneca, Bayer, Boehringer Ingelheim, Bristol Myers Squibb, Endo Laboratories, GSK, Ikenna Ogbaa/Lexicon Pharmaceutical, LASER Europe Limited, Lilly, Lundbeck, Novartis, Novo Nordisk Pharmaceuticals, Otsuka, Pfizer, Pierre Fabre Dermatologie, Roche, Sanofi, Takeda, Teva, UCB and ViiV Healthcare, outside the submitted work. The remaining authors (DBH, MMT, MEP) have declared that no competing interests exist.

Patient consent Not human subjects research, no consent form used

Ethics approval This study was declared exempt from review by the institutional review boards of the University of Pennsylvania (818082) and Rutgers Biomedical and Health Sciences (Pro20150002631). This study was approved by The Health Improvement Network's scientific review committee (139043).

Provenance and peer review Not commissioned; externally peer reviewed.

Data sharing statement Technical appendix and statistical code are available from the corresponding author upon request.

Open Access This is an Open Access article distributed in accordance with the Creative Commons Attribution Non Commercial (CC BY-NC 4.0) license, which permits others to distribute, remix, adapt, build upon this work non-commercially, and license their derivative works on different terms, provided the original work is properly cited and the use is non-commercial. See: http://creativecommons.org/ licenses/by-nc/4.0/

(c) Article author(s) (or their employer(s) unless otherwise stated in the text of the article) 2017. All rights reserved. No commercial use is permitted unless otherwise expressly granted.

\section{REFERENCES}

1. Heimann WG, Freiberger RH. Avascular necrosis of the femoral and humeral heads after high-dosage corticosteroid therapy. $N$ Engl J Med 1960;263:672-5.

2. Lafforgue P. Pathophysiology and natural history of avascular necrosis of bone. Joint Bone Spine 2006;73:500-7.

3. Gladman DD, Chaudhry-Ahluwalia V, Ibañez D, et al. Outcomes of symptomatic osteonecrosis in 95 patients with systemic lupus erythematosus. J Rheumatol 2001;28:2226-9.

4. Felson DT, Anderson JJ. Across-study evaluation of association between steroid dose and bolus steroids and avascular necrosis of bone. Lancet 1987;1:902-6.

5. Powell C, Chang C, Naguwa SM, et al. Steroid induced osteonecrosis: an analysis of steroid dosing risk. Autoimmun Rev 2010;9:721-43.
6. Dilisio MF. Osteonecrosis following short-term, low-dose oral corticosteroids: a population-based study of 24 million patients. Orthopedics 2014;37:e631-e636.

7. Cooper C, Steinbuch M, Stevenson R, et al. The epidemiology of osteonecrosis: findings from the GPRD and THIN databases in the UK. Osteoporos Int 2010;21:569-77.

8. Zhao DW, Yu M, Hu K, et al. Prevalence of nontraumatic osteonecrosis of the femoral head and its associated risk factors in the Chinese population: results from a nationally representative survey. Chin Med J 2015;128:2843-50.

9. Cui L, Zhuang Q, Lin J, et al. Multicentric epidemiologic study on six thousand three hundred and ninety five cases of femoral head osteonecrosis in China. Int Orthop 2016;40:267-76.

10. Tsampalieros A, Lam CK, Spencer JC, et al. Long-term inflammation and glucocorticoid therapy impair skeletal modeling during growth in childhood Crohn disease. J Clin Endocrinol Metab 2013;98:3438-45.

11. Perry DC, Bruce CE, Pope D, et al. Comorbidities in Perthes' disease: a case control study using the General Practice Research database. J Bone Joint Surg Br 2012;94:1684-9.

12. te Winkel ML, Pieters R, Hop WC, et al. Prospective study on incidence, risk factors, and long-term outcome of osteonecrosis in pediatric acute lymphoblastic leukemia. J Clin Oncol 2011;29:4143-50.

13. Nakamura J, Saisu T, Yamashita K, et al. Age at time of corticosteroid administration is a risk factor for osteonecrosis in pediatric patients with systemic lupus erythematosus: a prospective magnetic resonance imaging study. Arthritis Rheum 2010;62:3248-15

14. Blak BT, Thompson M, Dattani H, et al. Generalisability of the Health Improvement Network (THIN) database: demographics, chronic disease prevalence and mortality rates. Inform Prim Care 2011;19:251-5.

15. Lewis JD, Schinnar R, Bilker WB, et al. Validation studies of the health improvement network (THIN) database for pharmacoepidemiology research. Pharmacoepidemiol Drug Saf 2007;16:393-401.

16. Fardet L, Petersen I, Nazareth I. Prevalence of long-term oral glucocorticoid prescriptions in the UK over the past 20 years. Rheumatology 2011;50:1982-90.

17. Hollowell J. The General Practice Research Database: quality of morbidity data. Popul Trends 1997;87:36-40.

18. Lewis JD, Brensinger C, Bilker WB, et al. Validity and completeness of the General Practice Research Database for studies of inflammatory bowel disease. Pharmacoepidemiol Drug Saf 2002;11:211-8

19. Ogdie A, Langan S, Love T, et al. Prevalence and treatment patterns of psoriatic arthritis in the UK. Rheumatology 2013;52:568-75.

20. Seminara NM, Abuabara K, Shin DB, et al. Validity of the Health Improvement Network (THIN) for the study of psoriasis. $\mathrm{Br} J$ Dermatol 2011;164:no-9.

21. Somers EC, Thomas SL, Smeeth L, et al. Incidence of systemic lupus erythematosus in the United Kingdom, 1990-1999. Arthritis Rheum 2007;57:612-8.

22. Thomas SL, Edwards CJ, Smeeth L, et al. How accurate are diagnoses for rheumatoid arthritis and juvenile idiopathic arthritis in the general practice research database? Arthritis Rheum 2008;59:1314-21.

23. Lewis JD, Bilker WB, Weinstein RB, et al. The relationship between time since registration and measured incidence rates in the General Practice Research Database. Pharmacoepidemiol Drug Saf 2005;14:443-51.

24. Kadan-Lottick NS, Dinu I, Wasilewski-Masker K, et al. Osteonecrosis in adult survivors of childhood cancer: a report from the childhood cancer survivor study. J Clin Oncol 2008;26:3038-45.

25. Perry DC, Bruce CE, Pope D, et al. Legg-Calvé-Perthes disease in the UK: geographic and temporal trends in incidence reflecting differences in degree of deprivation in childhood. Arthritis Rheum 2012;64:1673-9.

26. Mattano LA, Sather HN, Trigg ME, et al. Osteonecrosis as a complication of treating acute lymphoblastic leukemia in children: a report from the Children's Cancer Group. J Clin Oncol 2000;18:3262-72.

27. Nakasian SS, Rassen JA, Franklin JM. Effects of expanding the lookback period to all available data in the assessment of covariates. Pharmacoepidemiol Drug Saf 2017.

28. D'Agostino RB, Lee ML, Belanger AJ, et al. Relation of pooled logistic regression to time dependent cox regression analysis: the Framingham Heart Study. Stat Med 1990;9:1501-15.

29. Cupples LA, D'Agostino RB, Anderson K, et al. Comparison of baseline and repeated measure covariate techniques in the Framingham Heart Study. Stat Med 1988;7(1-2):205-18. 
30. Haynes K, Bilker WB, Tenhave TR, et al. Temporal and within practice variability in the health improvement network. Pharmacoepidemiol Drug Saf 2011;20:n/a-55.

31. Bursac Z, Gauss CH, Williams DK, et al. Purposeful selection of variables in logistic regression. Source Code Biol Med 2008;3:17.

32. Sainani KL. Multivariate regression: the pitfalls of automated variable selection. Pm R 2013;5:791-4.

33. Fisher RL. An epidemiological study of Legg-Perthes disease. J Bone Joint Surg Am 1972;54:769-78.

34. Korn EL, Graubard BI. Analysis of health surveys. New York: Wiley, 1999.

35. Davidson R, MacKinnon JG. Bootstrap tests: how many bootstraps? Econom Rev 2000;19:55-68.

36. Gerber JS, Prasad PA, Fiks AG, et al. Effect of an outpatient antimicrobial stewardship intervention on broad-spectrum antibiotic prescribing by primary care pediatricians: a randomized trial. JAMA 2013;309:2345-52

37. Sterne JA, White IR, Carlin JB, et al. Multiple imputation for missing data in epidemiological and clinical research: potential and pitfalls. BMJ 2009;338:b2393-b93.

38. Schneeweiss S. Sensitivity analysis and external adjustment for unmeasured confounders in epidemiologic database studies of therapeutics. Pharmacoepidemiol Drug Saf 2006; 15:291-303.

39. National Center for Health Statistics. Health, United States, 2013:special feature on prescription drugs. Hyattsville, MD, 2014
40. Brunner HI, Silverman ED, To T, et al. Risk factors for damage in childhood-onset systemic lupus erythematosus: cumulative disease activity and medication use predict disease damage. Arthritis Rheum 2002;46:436-44.

41. Kaste SC. Skeletal toxicities of treatment in children with cancer Pediatr Blood Cancer 2008;50(2 Suppl):469-73.

42. Lilleby V. Bone status in juvenile systemic lupus erythematosus. Lupus 2007;16:580-6.

43. Hwang YG, Saag K. The safety of low-dose glucocorticoids in rheumatic diseases: results from observational studies. Neuroimmunomodulation 2015;22(1-2):72-82.

44. van Staa TP, Leufkens HG, Abenhaim L, et al. Oral corticosteroids and fracture risk: relationship to daily and cumulative doses. Rheumatology 2000;39:1383-9.

45. Smith FE, Sweet DE, Brunner CM, et al. Avascular necrosis in SLE. an apparent predilection for young patients. Ann Rheum Dis 1976;35:227-32.

46. Zizic TM, Marcoux C, Hungerford DS, et al. Corticosteroid therapy associated with ischemic necrosis of bone in systemic lupus erythematosus. Am J Med 1985;79:596-604.

47. Abeles M, Urman JD, Rothfield NF, et al. Aseptic necrosis of bone in systemic lupus erythematosus. Relationship to corticosteroid therapy. Arch Intern Med 1978;138:750-4.

48. Shigemura T, Nakamura J, Kishida S, et al. Incidence of osteonecrosis associated with corticosteroid therapy among different underlying diseases: prospective MRI study. Rheumatology 2011;50:2023-8. 